\title{
Diagnostic value of sural nerve biopsy in chronic inflammatory demyelinating polyneuropathy
}

\author{
D S M Molenaar, $M$ Vermeulen, $R$ de Haan
}

\begin{abstract}
Objective-To investigate the additional diagnostic value of sural nerve biopsy of 64 patients in whom chronic inflammatory demyelinating polyneuropathy (CIDP) was considered, as sural nerve biopsy is recommended in the research criteria of an ad hoc subcommittee to diagnose CIDP.
\end{abstract}

Methods-Firstly, the additional diagnostic value of sural nerve biopsy was analysed with multivariate logistic regression. Six clinical features (remitting course, symmetric sensorimotor neuropathy in arms and legs, areflexia, raised CSF protein concentration, nerve conduction studies consistent with demyelination, and absence of comorbidity or relevant laboratory abnormalities) were entered into a logistic model. Afterwards, all significant features identified from this model, as well as the results of sural nerve biopsy were forced into a second logistic model. Secondly, the diagnostic performance of a neurologist experienced in diagnosis of peripheral nerve disorders was studied by receiver operating characteristics (ROC) curve analysis.

Results-The results of the first logistic analysis showed that CSF protein concentration $>1 \mathrm{~g} / 1$ (odds ratio $(O R)=38.5)$ and neurophysiological studies consistent with demyelination $(O R=51.7)$ were strong predictors of CIDP. When forcing the significant features and the sural nerve biopsy data into the model, an independent predictive value of sural nerve biopsy could not be found. The neurologist was able to discriminate patients with and without CIDP (area under the curve $(A U C)=0.95)$. His diagnostic performance did not improve significantly by offering him the results of sural nerve biopsy.

Conclusion-Any additional diagnostic value of sural nerve biopsy in the diagnosis of CIDP could not be shown.

(F Neurol Neurosurg Psychiatry 1998;64:84-89)

Keywords: chronic inflammatory demyelinating polyneuropathy; sural nerve biopsy

Sural nerve biopsy is considered to be a valuable method for establishing the cause of peripheral neuropathies in specific circumstances, if it is evaluated by a neuropathologist experienced in modern techniques. ${ }^{1}$ It is indicated in a few patients with peripheral neuropathy and should be done only after careful clinical, laboratory, and neurophysiological evaluation. ${ }^{2}$ Whether suspected chronic inflammatory demyelinating polyneuropathy (CIDP) is an indication for sural nerve biopsy studies remains inconclusive from reports in the literature. Argov et al, for instance, suggest that sural nerve biopsy should be reserved for patients with a clinical picture of polyneuropathy and electrodiagnostic criteria suggestive of demyelination. ${ }^{3}$ Asbury, however, recommends the use of sural nerve biopsy only in cases of a clinical picture of mononeuritis multiplex, enlarged nerves at palpation, or for establishing diagnosis in genetically determined paediatric disorders. ${ }^{4}$ Sural nerve biopsy studies have been reported in several series of patients with CIDP. The abnormalities found are often not specific for CIDP. ${ }^{5}$ On the other hand, sural nerve biopsy is a requisite for diagnosis of "definite" CIDP according to the research criteria for diagnosis of CIDP of the ad hoc subcommittee, published in $1990 .^{6}$

Other tests to diagnose CIDP include lumbar puncture, withdrawal of blood, and neurophysiological tests. Sural nerve biopsy is the most invasive procedure of all these procedures and should be considered after the results of the other tests are known. ${ }^{7-10}$ However, little is known about the diagnostic properties of sural nerve biopsy in CIDP. Therefore, the objectives of this study were (1) to investigate the objective additional diagnostic value of sural nerve biopsy in CIDP, given the results of the clinical picture, and laboratory and neurophysiological tests, and (2) to assess to what extent the diagnostic performance of an experienced neurologist was influenced by the results of sural nerve biopsy.

\section{Methods}

Medical records were reviewed of patients from seven university hospitals in The Netherlands who underwent a sural nerve biopsy between 1989 and 1994. Included in the study were patients in whom CIDP was considered in the differential diagnosis before sural nerve biopsy. Sural nerve biopsies were processed and assessed according to standard techniques, including teased fibre preparations. ${ }^{2}$ Electron microscopy was carried out when the neuropathologist considered that this was necessary. Excluded were patients with an appropriate history of drug or toxic exposure known to cause peripheral neuropathy, with a known family history of peripheral neuropathy, and with incomplete data on clinical signs and symptoms, blood tests, CSF tests, electrophysiological studies, biopsy studies, response to treatment, and clinical course after biopsy. 
ASSESSMENT OF CLINICAL FEATURES AND SURAL NERVE BIOPSY

Six clinical features, from the period before sural nerve biopsy was performed, were extracted from the medical records. The features were the presence of (1) relapsing course; (2) symmetric sensorimotor neuropathy in arms and legs; (3) areflexia of all four limbs; (4) raised CSF protein concentration (moderately $(0.5-1.0 \mathrm{~g} / \mathrm{l})$ and highly $(>1.0$ $\mathrm{g} / \mathrm{l}$ ) raised); (5) neurophysiological studies consistent with CIDP, and (6) relevant comorbidity or relevant abnormalities in laboratory tests that were sufficient to explain the occurrence of peripheral neuropathy. Neurophysiological studies were considered consistent with CIDP if there was a non-uniform pattern of abnormalities and if three of the following four abnormalities were shown: (a) slowed nerve conduction velocity, defined as less than $75 \%$ of the lower limit of normal, of at least one motor nerve in the arms; $(b)$ partial conduction block in one or more motor nerves, defined as difference of at least $30 \%$ in compound muscle action potential (peak to peak) recorded after stimulation at proximal and distal segments and provided that the potential after distal stimulation is more than $1.0 \mathrm{mV}$ peak to peak; (c) prolonged distal latency of more than $130 \%$ of the upper limit of normal in one or more motor nerves in the arms; and (d) prolonged $F$ wave of more than $130 \%$ of the upper limit of normal in one or more motor nerves. The abnormalities were modified from the neurophysiological criteria of Albers and Kelly. ${ }^{11}$ Instead of the presence of decreased nerve conduction velocity in at least two motor nerves, irrespective of arm or leg nerves, we used its presence in at least one motor nerve in the arm as the criterion. For prolonged distal latency we also used presence in at least one motor nerve in the arms as a criterion instead of its presence in at least two motor nerves, irrespective of arm or leg nerves.

The reports of sural nerve biopsies were reviewed in a random order without knowledge of the patient's clinical data. The conclusions of the neuropathologist were classified as: (1) Not consistent with CIDP - that is, either with abnormalities suggestive of another diagnosis or with no or no specific abnormalities; and (2) consistent with CIDP - that is, with demyelination or with both demyelination and cellular infiltrates.

ASSESSMENT OF THE DIAGNOSTIC BEHAVIOUR OF A NEUROLOGIST

Firstly, a neurologist experienced in diagnosis of peripheral nerve disorders (MV) reviewed the clinical data of each patient from the period before sural nerve biopsy and recorded the following clinical diagnoses: No CIDP; CIDP with low probability; CIDP with moderate probability; CIDP with high probability; and almost definite CIDP. The within observer reliability of his diagnostic behaviour was assessed within a subsample of the patients at least two months after he had recorded the first diagnosis.
Two months after the first assessment without the results of sural nerve biopsy, the same clinical data were presented to the same neurologist in a randomly selected order, but this time in combination with the conclusions of the biopsy reports. Again, the neurologist recorded a diagnosis as mentioned previously.

APPROACH TO GOLD STANDARD

Follow up data of all patients, consisting of clinical course after biopsy, response to treatment, and diagnosis established by the treating neurologist, were screened. The final diagnosis-(1) a diagnosis other than CIDP or (2) CIDP - was based on a relapsing, remitting course after biopsy or a beneficial response to treatment with either intravenous immunoglobulin (IVIg), plasma exchange, or prednisone in patients with clinical signs and symptoms of CIDP. When these data were unequivocal, the diagnosis established by the neurologist who treated the patient was decisive.

\section{Analysis}

The patients' demographic characteristics and clinical features were analysed with descriptive statistics.

ANALYSIS OF OBJECTIVE DIAGNOSTIC VALUE OF

THE CLINICAL FEATURES AND SURAL NERVE BIOPSY

For each of the six clinical features, as well as the biopsy, we calculated sensitivity (the proportion of patients with CIDP who had a positive test result), specificity (the proportion of patients who did not have CIDP and had a negative test result), and positive likelihood ratios (LRs) for CIDP. A positive LR refers to the odds of a positive diagnostic test result in a patient with CIDP compared with a patient without CIDP.

Next, we entered the six clinical features into a logistic model to identify all significant $(p \leqslant 0.20)$ features. These significant features, as well as the conclusions of sural nerve biopsy reports were additionally forced into a second logistic model. The effect sizes of both models were expressed as odds ratios. The odds ratio approximates how much more likely (or unlikely) CIDP is among patients with the characteristic of interest than among patients without that characteristic.

ANALYSIS OF DIAGNOSTIC BEHAVIOUR OF A NEUROLOGIST

The diagnostic performance of the neurologist himself in terms of within observer reliability was assessed with weighted Kappa statistics $(\kappa)$, which expresses the proportion of agreement beyond chance. Values for $\kappa$ can be arbitrarily interpreted as poor $(\kappa<0.20)$, fair $(\kappa=0.21-0.40)$, moderate $(\kappa=0.410 .60)$, substantial $(\kappa=0.61-0.80)$, or almost perfect $(\kappa=0.81-1.00) .^{12}$

The diagnostic behaviour of the neurologist was studied by receiver operating characteristics (ROC) curve analyses. ${ }^{13}$ In this case, the ROC curve depicts how successfully the neurologist was able to discriminate patients with and without CIDP by plotting the sensitivity of his 
Table 1 Diagnoses other than CIDP in 41 patients

\begin{tabular}{lc}
\hline Diagnosis & Patients $(n)$ \\
\hline Vasculitis neuropathy & 6 \\
Neuropathy with monoclonal gammopathy of undetermined significance & 5 \\
Multiple sclerosis and neuropathy & 3 \\
Inherited neuropathy & 2 \\
Neuropathy with non-Hodgkin's lymphoma & 1 \\
Myotonic dystrophy with neuropathy & 1 \\
Lyme neuropathy & 1 \\
Alcohol neuropathy & 2 \\
Guillain-Barré syndrome & 1 \\
Neuropathy of unknown aetiology: & 7 \\
$\quad$ Chronic idiopathic axonal polyneuropathy ${ }^{18}$ & 11 \\
$\quad$ Not classified & 1 \\
No neuropathy & \\
\hline
\end{tabular}

diagnostic performance against 1-specificity at five possible cut off points. These cut off points reflected the various CIDP probabilities (no CIDP, low, moderate, or high probability of CIDP, and almost certain CIDP) as assessed by the neurologist. ROC analyses of the neurologist's diagnostic behaviour were carried out with and without his knowledge of the results of sural nerve biopsy. To compare the overall accuracy of the neurologist's diagnostic behaviour we also calculated the areas under the ROC curve (AUCs) and their 95\% confidence intervals (95\% CIs). The AUC represents the probability that a random pair of patients will be correctly classified by the neurologist as having or not having CIDP. A value of 0.50 is obtained when the physician performs not better than chance, and a value of 1.0 means perfect accuracy.

\section{Results}

DESCRIPTION OF DEMOGRAPHIC

CHARACTERISTICS OF THE PATIENTS

Sixty four patients fulfilled the inclusion criteria listed in the methods section. There were 37 males and 27 females, aged 4-80 (median 54.0) years. Onset of disease before biopsy ranged from one month to 11 years (median 11.5 months). The median follow up period of all patients was three months (range 0.5-67 months) after the biopsy had been taken.

DESCRIPTION OF FOLLOW UP DATA AND

CONCLUSIONS OF SURAL NERVE BIOPSY REPORTS

According to the approach to the gold standard 23 patients had CIDP and 41 had another diagnosis. Nineteen of 23 patients with CIDP had a beneficial response to treatment with IVIg, plasma exchange, or prednisone. One patient with CIDP improved spontaneously and three patients did not improve after immunomodulating treatment. These patients were diagnosed as CIDP by the treating neurologist. Table 1 shows the other diagnoses.

Table 2 Clinical features and sural nerve biopsy in 23 patients with CIDP and 41 patients with another diagnosis: sensitivity, specificity, and positive LR for the diagnosis of CIDP

\begin{tabular}{llll}
\hline Clinical feature & Sensitivity & Specificity & $\begin{array}{l}\text { Positive LR } \\
\text { for CIDP }\end{array}$ \\
\hline (1) Remitting course & 0.39 & 0.88 & 3.21 \\
(2) Symmetric sensorimotor neuropathy & 0.70 & 0.78 & 3.17 \\
(3) Areflexia & 0.52 & 0.80 & 2.67 \\
(4) Raised CSF protein concentration $(>0.5 \mathrm{~g} / \mathrm{l})$ & 0.87 & 0.44 & 1.55 \\
$\quad$ Highly raised $(>1 \mathrm{~g} / 1)$ & 0.57 & 0.88 & 4.63 \\
(5) Neurophysiological studies consistent with & 0.87 & 0.85 & 5.94 \\
CIDP & & & \\
(6) Absence of comorbidity & 0.65 & 0.63 & 1.78 \\
(7) Sural nerve biopsy consistent with CIDP & 0.61 & 0.78 & 2.77 \\
\hline
\end{tabular}

Comorbidity, relevant laboratory changes, or relevant medical histories in eight patients with CIDP consisted of non-insulin dependent diabetes mellitus (one), low level paraprotein type IgG kappa (one), both paraprotein type IgG lambda and history of alcohol misuse (one), history of alcohol misuse only (one), pregnancy (one), history of carcinoma (two), and history of rheumatoid arthritis treated with gold (one). None were considered to be the cause of the neuropathy.

Teased fibre preparations were taken from all biopsy specimens. Histometry and electron microscopy were performed in $58 \%$ of the biopsies each. Nine patients with CIDP had sural nerve biopsies that were normal (one), showed axonal degeneration and regeneration (six), or were suggestive of vasculitis (two). Neither of the two biopsies suggestive of vasculitis were studied with electron microscopy, but histometry was performed in the biopsy of the first patient. This patient had a chronic progressive symmetric sensorimotor neuropathy, areflexia, raised CSF protein concentration $(>1 \mathrm{~g} / \mathrm{l})$, and neurophysiological studies consistent with demyelination. She was pregnant at the onset of the neuropathy. The evidence for CIDP seemed strong by neurological signs and symptoms, CSF protein concentration, and neurophysiological data and therefore the results of sural nerve biopsy were disregarded. Neurological signs and symptoms did not improve after IVIg but did improve after prednisone. The follow up period after biopsy was 20 months, in which the treating neurologist did not change the diagnosis of CIDP. The other patient with a biopsy suggestive of vasculitis had a symmetric, predominant sensory neuropathy, areflexia, raised CSF protein concentration, and physiological studies consistent with demyelination. $\mathrm{He}$ had a history of gastric carcinoma. After biopsy he was first diagnosed as having non-systemic vasculitis neuropathy. Treatment with high dose prednisone and cyclosporin was not effective. After cessation of cyclosporin, however, neurological signs and symptoms deteriorated. The treating neurologist reconsidered the diagnosis non-systemic vasculitis and diagnosed CIDP. After follow up of 67 months, the diagnosis of CIDP was not changed.

In the group of patients with a diagnosis other than CIDP, biopsy results of nine patients were consistent with CIDP. Two of these patients had multiple sclerosis and neuropathy. These patients had no symmetric sensorimotor neuropathy of the arms and legs, no areflexia, and physiological studies were not consistent with demyelination. Their biopsies showed demyelination. Clinical evidence did not seem strong enough to diagnose the patients as having CIDP associated with multiple sclerosis. Other diagnoses after follow up were Guillain-Barré syndrome, polyneuropathy with monoclonal gammopathy of IgM type, neuropathy with non-Hodgkin's lymphoma, and neuropathy with Lyme's disease, each in one patient, and three patients were diagnosed as having neuropathy of unknown aetiology. The neurologists who followed up the last three 
Table 3 Logistic regression models to predict CIDP with six clinical features and sural nerve biopsy in a group of 64 patients in whom CIDP was considered in the differential diagnosis

\begin{tabular}{|c|c|c|c|c|}
\hline \multirow[b]{2}{*}{ Clinical feature } & \multicolumn{2}{|c|}{$\begin{array}{l}\text { Model 1: after entering } \\
\text { six clinical features }\end{array}$} & \multicolumn{2}{|c|}{$\begin{array}{l}\text { Model 2: after entering the significant }(p \leqslant 0.20) \\
\text { clinical features, as identified in the first model, and } \\
\text { sural nerve biopsy }\end{array}$} \\
\hline & $O R$ & P Value ${ }^{*}$ & $O R$ & $P$ Value ${ }^{\star}$ \\
\hline (1) Remitting course & 1.6 & 0.70 & - & - \\
\hline (2) Symmetric sensorimotor neuropathy & 5.3 & 0.14 & 5.0 & 0.15 \\
\hline (3) Areflexia & 1.3 & 0.80 & - & - \\
\hline (4) Raised CSF protein concentration $(0.5-1.0 \mathrm{~g} / \mathrm{l})$ & 7.7 & 0.13 & 9.0 & 0.11 \\
\hline Highly raised $(>1 \mathrm{~g} / \mathrm{l})$ & 38.5 & 0.01 & 58.1 & 0.01 \\
\hline $\begin{array}{l}\text { (5) Neurophysiological studies consistent with } \\
\text { CIDP }\end{array}$ & 51.7 & 0.003 & 61.8 & 0.002 \\
\hline (6) Absence of comorbidity & 31.5 & 0.03 & 36.6 & 0.02 \\
\hline (7) Sural nerve biopsy consistent with CIDP & - & - & 2.2 & 0.42 \\
\hline
\end{tabular}

Dashes indicate that these factors were not entered into the model

${ }^{\star}$ Calculated with Wald statistics.

patients did not consider immunomodulating treatment. Seven patients in this group with another diagnosis than CIDP had sural nerve biopsies without abnormalities and 18 patients had sural nerve biopsies with signs of axonal degeneration and regeneration. Other diagnoses suggested by biopsy were vasculitis (six), hereditary motor and sensory neuropathy (HMSN) (one), and paraprotein neuropathy with IgM deposits (one).

OBJECTIVE DIAGNOSTIC VALUE OF SIX CLINICAL FEATURES AND SURAL NERVE BIOPSY

Table 2 shows the sensitivity, specificity, and positive LR for CIDP of the six clinical features and sural nerve biopsy.

Four of the six clinical features were more specific than sensitive, as was sural nerve biopsy. Relatively high sensitivity rates were seen in the presence of raised CSF protein concentration and neurophysiological studies consistent with CIDP. A relatively high rate of both sensitivity and specificity was found in neurophysiological studies consistent with CIDP. Our criteria required the demonstration of slowed motor nerve conduction velocities in at least one motor nerve of the arms; if these were present, the values were also abnormal in motor nerves of the legs. The highest positive LRs for CIDP were seen in highly raised CSF protein concentration and neurophysiological studies consistent with CIDP.

Table 3 shows the results of two logistic regression models. In the first model all six clinical features were entered. Highly raised CSF protein concentrations, neurophysiological studies consistent with CIDP, and, not surprisingly, absence of comorbidity were strong predictors of CIDP. The significant clinical features $(p \leqslant 0.20)$ as identified from the first logistic model were forced into the second model, as were the conclusions of sural nerve biopsy reports. The same clinical features turned out to be important predictive factors. An independent predictive ability of the sural nerve biopsy could not be shown. In other words, when adjusting for the important clinical features, we could not show that patients with sural nerve biopsy consistent with CIDP were more likely to have CIDP than patients with a negative sural nerve biopsy.
DIAGNOSTIC BEHAVIOUR OF THE NEUROLOGIST The diagnostic performance of the neurologist in terms of within observer reliability based on a subsample of 24 patients was $\kappa=0.92(95 \%$ CI $0.85-0.99)$. The figure shows the results of the neurologists's diagnostic behaviour. The neurologist was able to discriminate patients with and without CIDP (AUC $=0.95 ; 95 \%$ CI 0.90-1.00). His diagnostic performance was not improved any further by offering him additional information about sural nerve biopsy (AUC $=0.95 ; 95 \%$ CI 0.90-1.00).

As a consequence of the biopsy reports, the neurologist who reviewed the medical records changed his decision in five patients. The first three patients had CIDP and the last two patients had another diagnosis. Histometry was performed on the biopsies of all three patients with CIDP. Patient I was diagnosed as having almost definite CIDP before biopsy. After biopsy, which showed some abnormalities suggestive of vasculitis, the neurologist changed the diagnosis into moderate probability of CIDP. The evidence for CIDP seemed so strong that the neurologist did not change his diagnosis into no CIDP. Patient II was diagnosed as having highly probable CIDP before biopsy. The biopsy, which was also analysed with electron microscopy showed mixed demyelinating and axonal changes and inflammatory infiltrates, which made the neurologist decide to diagnose him as having definite CIDP. Patient III was diagnosed as having CIDP with moderate probability before biopsy and as having CIDP with high probability after biopsy, which had shown mixed demyelinating and axonal changes. Patient IV was diagnosed as having CIDP with moderate probability before biopsy and as having CIDP with low probability after biopsy, which had shown axonal degeneration. Patient $V$ was first diagnosed as having low probability of CIDP. The biopsy, which was also analysed with electron microscopy, showed features of vasculitis, which made the neurologist change the diagnosis into no CIDP.

\section{Discussion}

In this study we investigated the additional diagnostic value of the most invasive diagnostic procedure for the diagnosis of CIDP - sural nerve 


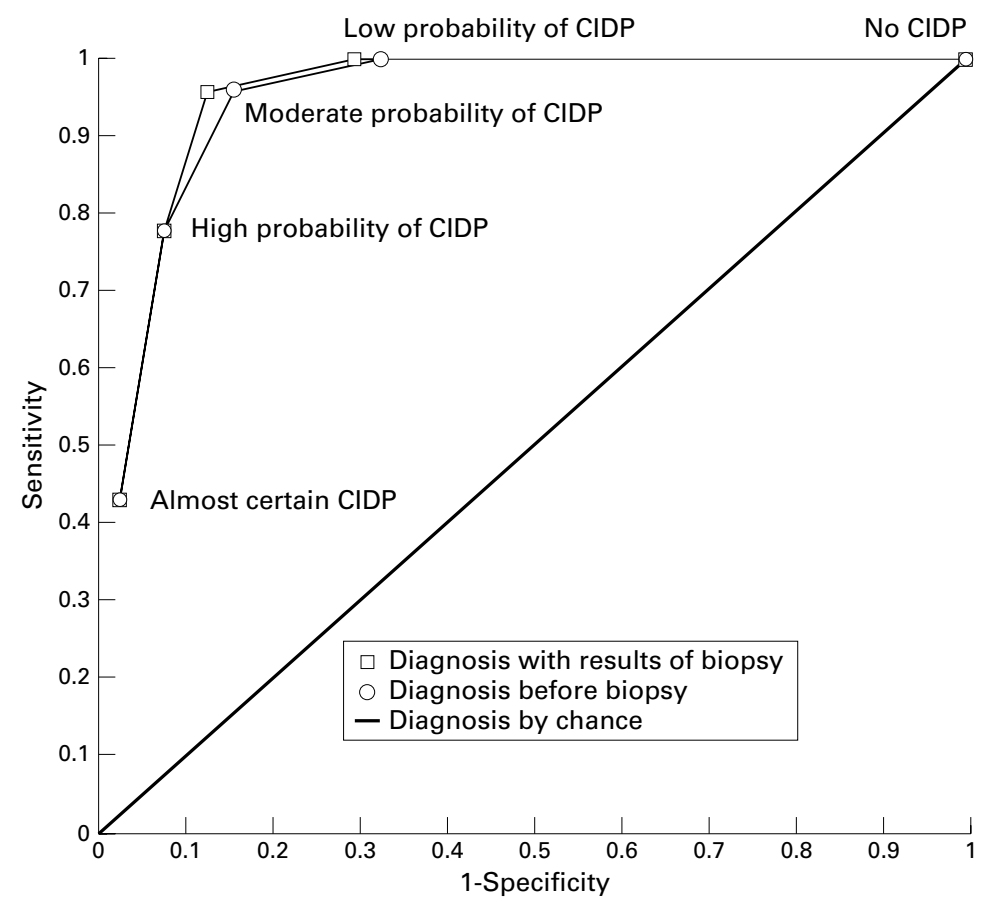

ROC curves for diagnosis recorded by an experienced neurologist after review of the clinical data before sural nerve biopsy, and diagnosis recorded after review of both clinical data and results of sural nerve biopsy.

biopsy-when the results of less invasive tests such as medical history, course and distribution of neurological signs and symptoms, and results of blood tests, CSF protein, and neurophysiological studies were already known.

In the first part of this study, we analysed the objective diagnostic properties of six clinical features and of sural nerve biopsy consistent with CIDP. Neurophysiological studies consistent with demyelination, highly raised CSF protein concentrations and, not surprisingly, absence of comorbidity, were strong predictors for CIDP, whereas this could not be shown for sural nerve biopsy consistent with CIDP.

The objective of the second part of the study was to analyse the diagnostic behaviour of a neurologist experienced in diagnosing peripheral neuropathies. After review of the biopsy reports the neurologist changed his diagnosis in only five of 64 patients. He was able to distinguish between patients with and without CIDP, irrespective of the biopsy data. These results confirm the absence of additional diagnostic value of sural nerve biopsy for diagnosis of CIDP as was statistically shown in the first part of our study.

In all cases the treating neurologist had asked the neuropathologist whether the biopsy was consistent with CIDP. The low diagnostic value of sural nerve biopsy as shown in this study can, therefore, not be explained by the lack of attention in searching for features of inflammatory demyelinating neuropathy. Neither can the results be explained by lack of knowledge of the features consistent with CIDP or the use of inappropriate techniques. All biopsies were investigated after 1989, when the biopsy features and techniques to demonstrate inflammatory demyelinating neuropathies were widely known. ${ }^{14}$
It is difficult to compare the percentage of demyelination found in sural nerve biopsies in our group of patients with CIDP with that found in other groups of patients with the disease. ${ }^{15}{ }^{16}$ We relied on the conclusion of the neuropathologist, and found that $61 \%$ of patients with CIDP had sural nerve biopsies with demyelination. Barohn et al reported predominantly demyelination in $48 \%$, mixed demyelination and axonal changes in $13 \%$, predominantly axonal changes in $21 \%$, and no abnormalities in $18 \%$ of sural nerve biopsies of patients with CIDP. ${ }^{17}$ Krendel et al reported predominantly demyelination in $50 \%$ of sural nerve biopsies of 14 patients with CIDP. ${ }^{5}$

This study was not performed to investigate sensitivity and specificity of sural nerve biopsy in CIDP. In fact, we specifically focused on the additional diagnostic value of sural nerve biopsy in CIDP, which means that we investigated whether sural nerve biopsy increases the probability of the diagnosis CIDP. The design of the study simulated clinical practice, as sural nerve biopsy is usually performed when all other test results are known. Both objective and subjective analyses showed that sural nerve biopsy is a weak diagnostic test. Therefore, sural nerve biopsy is not helpful in confirming the diagnosis of CIDP irrespective of whether there is considerable doubt or almost certainty about the diagnosis of CIDP before biopsy. We conclude that there is no reason to include sural nerve biopsy in research criteria of CIDP, and that sural nerve biopsy has no value in clinical practice to confirm the diagnosis CIDP before embarking on immunosuppressive treatment.

We thank AAWM Gabreëls-Festen for transposing the data and critical reading of the manuscript. We also thank PA van Doorn, AEJ de Jager, W Kamphorst, AR Wintzen, and JHJ Wokke for transposing their data.

$1 \mathrm{Mc}$ Leod JG. Investigation of peripheral neuropathy. $\mathcal{F} \mathrm{Neu}-$ rol Neurosurg Psychiatry 1995;58:274-83.

2 Dyck PJ, Giannini C, Lais A. Pathologic alterations of nerves. In: Dyck PJ, et al. Peripheral neuropathy. Philadelphia: WB Saunders, 1993:514-95.

3 Argov Z, Steiner I, Soffer D. The yield of sural nerve biopsy in the evaluation of peripheral neuropathies. Acta Neurol Scand 1989;79:243-5.

4 Asbury AK. Disorders of peripheral nerve. In: Asbury AK, ed. Diseases of the nervous system. Philadelphia: WB Saunders, 1986:321-36.

5 Krendel DA, Parks HP, Anthony DC, St.Clair MB, Graham DG. Sural nerve biopsy in chronic inflammatory demyelinating polyradiculoneuropathy. Muscle Nerve 1989;12:257-264. Research criteria for diagnosis of chronic inflammatory
demyelinating polyneuropathy (CIDP). Report from an Ad Hoc Subcommittee of the American Academy of Neurology AIDS Task Force. Neurology 1991;41:617-8.

7 Solders G. Discomfort after fascicular sural nerve biopsy. Acta Neurol Scand 1989;77:503-4.

8 Rappaport WD, Valente J, Hunter GC, et al. Clinical utilization and complications of sural nerve biopsy. Am f Surg 1993;166:252-6.

9 Neundörfer B, Grahmann F, Engelhardt A, Harte U. Postoperative effects and value of sural nerve biopsies: a retrospective study. Eur Neurol 1990;30:350-2.

$10 \mathrm{Oh}$ SJ. Diagnostic usefulness and limitations of the sural nerve biopsy. Yonsei Med $\mathcal{F}$ 1990;31:1-31.

11 Albers JW, Kelly JJ. Acquired inflammatory demyelinating polyneuropathy: clinical and electrodiagnostic features. polyneuropathy: clinical and

12 Altman DG. Some common problems in medical research. In: Altman DG, ed. Practical statistics for medical In: Altman DG, ed. Practical statistics for medica
research.London: Chapman and Hall, 1991:396-439.

13 The selection of diagnostic tests. In: Sackett DL, Haynes $\mathrm{RB}$, Guyatt GH, Tugwell P, eds. Clinical epidemiology: a basic science for clinical medicine. Boston: Little Brown, 1991:51-68

14 Dyck PJ, Karnes J, Lais A, Lofgren EP, Stevens JC. Pathologic alterations of the peripheral nervous system in humans. In: Dyck PJ, et al, eds. Peripheral neuropathy. Vol 1. 
Philadelphia: WB Saunders, 1984:769-870.

15 Dyck PJ, Lais AC, Michiya O, Bastron JA, Harud O, Groover RV. Chronic inflammatory polyradiculoneuropathy. ver RV. Chronic inflammator

16 McCombe PA, Pollard JD, McLeod JG. Chronic inflammatory demyelinating polyradiculoneuropathy. A clinical and electrophysiological study of 92 cases. Brain 1987;110: $1617-30$.
17 Barohn RJ, Kissel JT, Warmolts JR, Mendell JR. Chronic inflammatory demyelinating polyradiculoneuropathy. diagnostic criteria. Arch Neurol 1989;46:878-84.

18 Notermans NC, Wokke JHJ, Franssen H, et al. Chronic idiopathic polyneuropathy presenting in middle or old age: a clinical and electrophysiological study of 75 patients. $\mathcal{f}$ Neurol Neurosurg Psychiatry 1993;56:1066-71.

\section{NEUROLOGICAL STAMP}

\section{Charles Edouard Brown-Séquard (1818-94)}

Brown-Séquard, the son of an American sea captain and a French woman, was born on the island of Mauritius. Mauritius had formerly been French but it was British at the time of Brown-Séquard's birth, making him a British subject. He practised at varying times in Paris, Mauritius, London, and New York. He remained longer (four and a half years) at The National Hospital in London of which he was a founder physician, than at any other place or in any other post until 14 years later when he succeeded Claude Bernard in his final appointment as Professor of Medicine at the College de France. He took his medical degree in Paris in 1846 with a thesis on the study of reflex movements and the functions of the columns of the spinal cord.

Brown-Séquard is largely remembered for his work on the spinal cord, which culminated in his classic rendition of the symptoms manifest by hemisection of the cord (1850, 1851). After his initial studies he continued to investigate the mode and site of conduction of motor and sensory impulses in the spinal cord and together with Türck laid the foundation of the anatomy and function of the spinal cord. Brown-Séquard's second important contribution to neurology comprised studies on sympathetic control of the vasomotor mechanisms. In 1852 he gave a series of lectures in Philadelphia in which he described contraction of the blood vessels of the face after galvanic stimulation of the cervical sympathetic nerves. This discovery proved embarrassing to Claude Bernard, who later reported the same discovery and misinterpreted the findings, unaware of Brown-Séquard's work. A series of experiments (published 1856) demonstrated the connection between the excision of the adrenal glands and Addison's disease. It is for this work that he is probably best known.

In his studies of epilepsy (1856) he sought the cause and treatment for the disease and, in so doing, was among the first to advocate bromides in the treatment of epilepsy. By the 1860s Brown-Sequard was recognised as an authority and expert on all diseases of the nervous system. He continued experimenting until the end of his 77 th year. During his tenure of the Professorship at the College de France, he investigated the possibility of prolonging human life by the use of extracts prepared from the testes of different animals. This led to many abuses, the commercialisation of the products, and much unfavourable publicity. Brown-Séquard became convinced from his imperfect experiments that not only the thyroid but also the adrenal, pancreas, liver, spleen and kidneys had internal secretions that could be employed in the treatment of disease. The term internal secretions was not his own but the creation of Claude Bernard, but Brown-Séquard's work gave great stimulus to the continuing studies.

He was honoured philatelically by Mauritius, the country of his birth, on the centenary of his death in 1994 (Stanley Gibbons 903, Scott 786). 